\title{
Intrinsic optical gain of ultrathin silicon quantum wells from first-principles calculations
}

\author{
Yuji Suwa ${ }^{1}$ and Shin-ichi Saito ${ }^{2}$ \\ ${ }^{1}$ Advanced Research Laboratory, Hitachi, Ltd., Kokubunji, Tokyo 185-8601, Japan \\ ${ }^{2}$ Central Research Laboratory, Hitachi, Ltd., and SORST, JST, Kokubunji, Tokyo 185-8601, Japan
}

(Received 29 May 2009; published 24 June 2009)

\begin{abstract}
Optical gains of ultrathin $\mathrm{Si}(001)$ quantum wells are calculated from first principles, and found to be positive because of an intrinsic quantum confinement effect. The gain of the ultrathin silicon film is comparable to that of the bulk GaAs if the carrier density is large enough. The impact of surface structure of the silicon film on the efficiency of light emission is also investigated and we found that $\mathrm{SiO}_{2}$ crystal that forms a strainless connection with a $\mathrm{Si}(001)$ surface such as quartz enhances optical gain.
\end{abstract}

DOI: 10.1103/PhysRevB.79.233308

PACS number(s): 78.66.Db, 78.20.Bh, 78.60.Fi

Observations of light emissions from silicon nanostructures ${ }^{1}$ such as porous silicon, ${ }^{2}$ nanocrystals, ${ }^{3}$ super lattices, ${ }^{4}$ and quantum wells ${ }^{5}$ have attracted much attention because of their potential applications in silicon photonics. ${ }^{6}$ An efficient silicon light emitter would have a significant impact on electronics industry because it can be easily incorporated into the state-of-the-art silicon technology. A nanostructure is a key to such applications because the confinement of carriers into a nanostructure modifies the indirect bandgap structure of bulk silicon and enables direct optical transition. Therefore, now it is an important subject of nanoscience to find what structure of silicon is the best for light emission and how effective such an emission is.

One of the goals of the improvements of silicon light emitters is to realize a silicon laser. A necessary condition for that is to obtain a net optical gain by a silicon nanostructure. That is not easy because the losses often exceed the small gains. While Pavesi et al. ${ }^{3}$ reported that the silicon nanocrystal has a net optical gain, the origin of that gain is considered to be extrinsic, i.e., defects due to the oxide passivation. ${ }^{7}$ Therefore, whether intrinsic optical gains by silicon nanostructures are attainable or not is still an unsolved question.

Recently we succeeded observing electroluminescence from an ultrathin silicon film in which a lateral carrier injection is easily performed. ${ }^{8}$ In order to understand clearly the mechanism of light emission in this film and to find a key factor in increasing efficiency of the emission, we performed first-principle calculations and studied optical direct transitions in ultrathin silicon films. In this Brief Report, utilizing the results of those calculations, we discuss importance of surface structures for efficient light emission and predict intrinsic optical gains in ultrathin silicon films.

The calculations are based on density functional theory (DFT) and use generalized gradient approximation ${ }^{9}$ with plane-wave-based ultrasoft pseudopotentials. ${ }^{10,11}$ The energy cutoff was set at 20.25 Rydberg. The convergence criterion used for geometry optimization was that all forces acting on the atoms are less than $1 \times 10^{-3} \mathrm{H} /$ a.u. The optical matrix elements were calculated with the aid of core repair terms. ${ }^{12}$

The fundamental mechanism of light emission in a silicon film is briefly summarized in the following. By taking the form of a thin film with (001) surfaces, silicon crystal acquires a direct bandgap structure due to the valley projection. ${ }^{13}$ As an example, Fig. 1(a) shows the band structure of $\mathrm{Si}(001)$ film with seven atomic layers. Here, the atomic structure of silicon crystal is not relaxed and dangling bonds are terminated by hydrogen atoms. It is clear that the minimum of the lowest conduction band (LCB) appears at the $\Gamma$ point. This minimum does not exist in the bulk and enables direct transition in the film. Figure 1(a) also shows that another minimum of LCB at the M point is higher than that at $\Gamma$. Such a rise of LCB at M point in a quantum well is due to a lighter effective mass in the direction perpendicular to the surface compared to that at $\Gamma .{ }^{14}$ Without this effect, the bandgap structure of this film will be pseudodirect. Figure 1(b) shows the band structure of a silicon film with (111) surfaces. In this case, no minimum appears at $\Gamma$; thus, the film still has an indirect bandgap structure.

For efficient light emission, a direct bandgap structure is not sufficient; large optical dipole matrix elements between the valence bands (VB) and the conduction bands (CB) are also necessary. The atomic structure of the silicon surface is
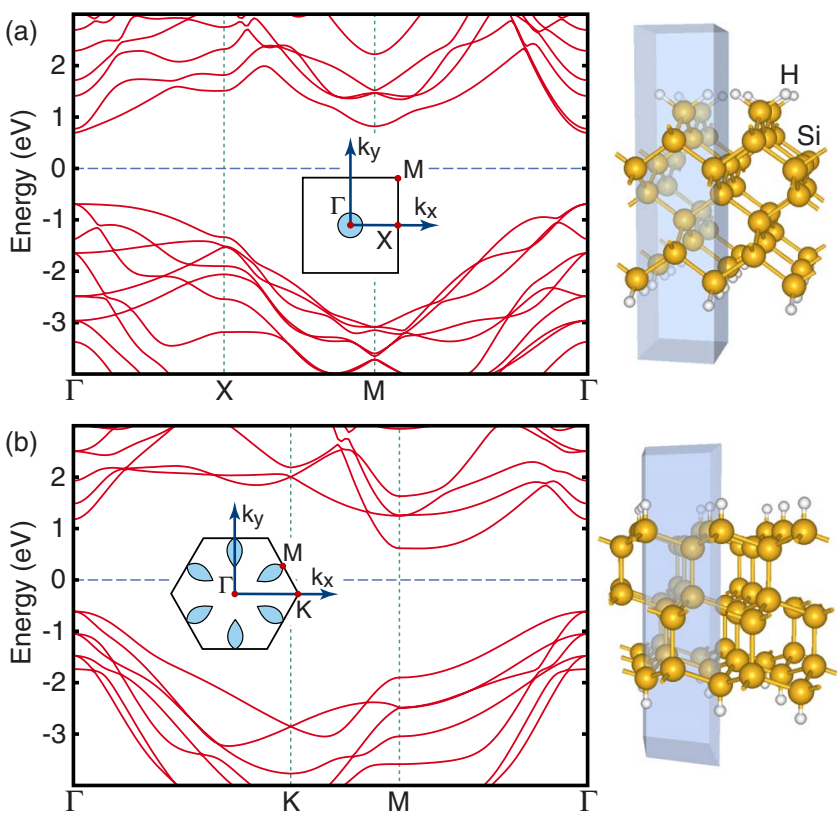

FIG. 1. (Color online) Band structures and atomic structures of $\mathrm{H}$-terminated (a) $\mathrm{Si}(001)$ film with seven atomic layers and (b) $\mathrm{Si}(111)$ film with six atomic layers, respectively. Translucent boxes are the unit cells for the calculations. Electron pockets due to the valley projection are shown in the insets. 

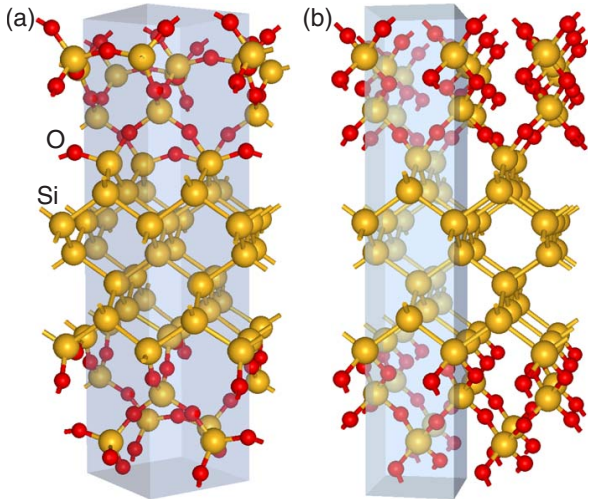

FIG. 2. (Color online) Atomic structures of silicon films sandwiched (a) between quartz layers and (b) between cristobalite layers. Translucent box in each figure shows the unit cell.

very important for determining such matrix elements, since it has a large influence on the wave function inside a silicon film with atomic-scale thickness.

Because the silicon film is thinned down by thermal oxidation in the experiment, ${ }^{8}$ we consider that a silicon layer is sandwiched between $\mathrm{SiO}_{2}$ layers. Although there are several theoretical studies, ${ }^{15,16}$ the atomic structure of the interface between silicon crystal and $\mathrm{SiO}_{2}$ prepared by thermal oxidation is not exactly known because it should depend on an experimental condition. We assume here that $\mathrm{SiO}_{2}$ has a crystalline structure at the interface, according to an experimental result that an ordered- $\mathrm{SiO}_{2}$ structure exists at the interface. ${ }^{17}$ This assumption is consistent with the fact that the surface of the silicon layer is atomically flat.

We calculated the matrix elements for three kinds of surface structures with various film thicknesses. One of the three structures is a hydrogen-terminated surface without geometry optimization as in the above-described band calculation. This example is expected to show a minimum surface effect. The other two structures are interfaces with quartz and cristobalite, as shown in Figs. 2(a) and 2(b), respectively. The interface with quartz is constructed by $\mathrm{Si}(001)$ and $\mathrm{SiO}_{2}(\overline{1} 100)$ faces using a $\mathrm{Si}(001)-\sqrt{2} \times \sqrt{2}$ unit cell as described in Ref. 18. The interface with cristobalite is constructed by inserting oxygen atoms between the $\mathrm{Si}-\mathrm{Si}$ bond of the bulk silicon by using a $\mathrm{Si}(001)-1 \times 1$ unit cell, in which $\mathrm{SiO}_{2}$ tetrahedrons are twisted around their $c$ axes as a result of geometry optimization. For both structures with $\mathrm{SiO}_{2}$, cell sizes along the $c$ axis (vertical direction) are optimized allowing relaxation of atomic positions, while the cell sizes in the horizontal direction are fixed to those of the silicon crystal. For these structures, first, we calculated optical dipole matrix elements $\left\langle\psi_{\mathrm{c}, k}|\boldsymbol{p}| \psi_{\mathrm{v}, k}\right\rangle$, where $\psi_{\mathrm{c}, k}$ and $\psi_{\mathrm{v}, k}$ are wave functions of $\mathrm{CB}$ and VB at $\boldsymbol{k}$, respectively. $\boldsymbol{p}$ is the momentum operator. The gain spectra at $300 \mathrm{~K}$ were then calculated using standard linear-response theory ${ }^{19}$ and normalized to the volume of the silicon quantum well.

Figure 3 shows optical gains for various thicknesses of the silicon film with three kinds of surface structures. Here, gap-crossing transitions between $\mathrm{CBs}$ and VBs only are taken into account. For each film, the peak value of the gain is obtained after the calculation of the energy-gain curve, as



FIG. 3. (Color online) Peak values of the gain spectrum for various thicknesses of silicon films. Transitions between CBs and VBs only are taken into account. Inset shows energy dependence of the gain of seven-atomic-layer silicon film between quartz layers. $n_{0}$ is equal to the carrier density of $4.8 \times 10^{20} \mathrm{~cm}^{-3}$.

shown in the inset. Here, contribution to the gain from each $k$ mesh point is smoothed by using Gaussian function with $1 / e$ width of $0.04 \mathrm{eV}$. Because optical transitions are almost in the direction parallel to the film, only a parallel component is shown. The densities of injected electrons and holes are assumed to be $4.8 \times 10^{20} \mathrm{~cm}^{-3}$. The overall tendency in Fig. 3 is similar to that of oscillator strength given in Ref. 20 calculated by the tight-binding method. It is clear that the films sandwiched between quartz give five to ten times higher gains than the other films over the wide range of film thicknesses. This result shows that the surface structure of the silicon film is an important factor in obtaining efficient electroluminescence.

To grasp how the surface structure affects the optical gain, we visualized the integrand of the optical matrix element in real space, i.e., $\boldsymbol{M}^{\mathrm{c}, \mathrm{v}}(\boldsymbol{r})=\psi_{\mathrm{c}, \boldsymbol{k}}^{*}(\boldsymbol{r}) \boldsymbol{p} \psi_{\mathrm{v}, \boldsymbol{k}}(\boldsymbol{r})$ is shown in Fig. 4. Note that the matrix element $\left\langle\psi_{\mathrm{c}, \boldsymbol{k}}^{*}|\boldsymbol{p}| \psi_{\mathrm{v}, \boldsymbol{k}}\right\rangle$ is its integral over the unit cell.

Figures 4(a) and 4(b) show the wave functions of the highest valence band (HVB) and LCB at $\Gamma$ in H-terminated silicon film with seven atomic layers. Figures 4(c) and 4(d) show the integrand $M$ between these two states parallel $\left(M_{\|}\right)$ and perpendicular $\left(M_{\perp}\right)$ to the surface, respectively. It can be clearly seen that $M_{\perp}$ vanishes when it is integrated over the unit cell, because the two contrasts of isosurfaces with opposite signs appear in equal proportion in each layer. On the other hand, $M_{\|}$is not canceled out at the integration completely, since $M_{\|}(\boldsymbol{r})$ changes its sign and amplitude by layers. This clearly illustrates the fact that the existence of the surface creates a finite optical matrix element. If there were no surfaces, i.e., the case of bulk crystal, $M_{\|}$will also be canceled out because the wave functions will be periodic along the $c$ axis. If the film was made of GaAs, famous as a lightemitting material even in the form of bulk crystal, $M(\boldsymbol{r})$ between HVB and LCB takes only one sign in the unit cell, as shown in Fig. 4(e); thus, its integral is always larger than those of silicon films.

To explain why the quartz structure at the interface favors 




FIG. 4. (Color online) (a) $\psi_{\mathrm{HVB}}$ and (b) $\psi_{\mathrm{LCB}}$ in H-terminated Si film with seven atomic layers. (c) $M_{\|}$and (d) $M_{\perp}$ between these two states, respectively. (e) $M$ between HVB and LCB in bulk GaAs. (f) $\psi_{\mathrm{HVB}}$ and $(\mathrm{g}) \psi_{\mathrm{LCB}}$ in 21-atomic-layer silicon film between quartz layers. (h) $M_{\|}$between (f) and (g). (i) Wave function of an excited state $\left(\psi_{\mathrm{CB}}\right)$. (j) $M_{\|}$between the states of (f) and (i). (k) $M_{\|}$between HVB and LCB in 21-atomic-layer silicon film between cristobalite layers. $\boldsymbol{k}$ point of each $\psi$ and $M$ is $\Gamma$.

optical transition, we show the wave functions and the integrands of 21-atomic-layer silicon film between quartz layers in Figs. 4(f)-4(j). Owing to the nature of $\psi_{\mathrm{HVB}}$ [Fig. 4(f)] showing a large amplitude near the upper surface, $M_{\|}(\boldsymbol{r})$ [Fig. 4(h)] between HVB and LCB has a significant amplitude near the surface. This leads to a large optical matrix element because the main contribution to the matrix element comes from the region near the surfaces. Asymmetry due to the surface is important in preventing canceling out of $M_{\|}$. It is therefore important that the wave function has a large amplitude near the surface. This effect can be seen more clearly, when the transition between HVB and a CB next to degenerate LCB is considered. Because the wave function of the CB [Fig. 4(i)] has a node at the center, it has a larger amplitude near the surface than that of the LCB [Fig. 4(g)]. As a result, $M_{\|}(\boldsymbol{r})[$ Fig. $4(\mathrm{j})]$ looks more one sided and gives a larger matrix element after integration. In fact, this transition contributes more to increasing the gain than the transition between HVB and LCB when the densities of injected carriers are large enough.

The reason $\psi_{\mathrm{HVB}}$ has a large amplitude near the surface in Fig. 4(f) is that the oxygen atoms at the surface of the quartz attract holes. On the other hand, the large strain of the atomic structure due to the mismatch of the lattice constant counteracts that effect at the surface of the cristobalite. $M_{\|}(\boldsymbol{r})$ of the silicon film between cristobalite layers is thus small at the surface as shown in Fig. 4(k). A hydrogen atom does not have such an ability to attract holes. If the wave function is attracted to the surface, its amplitude at the surface remains large even if the film is thickened, leading to a large optical matrix element. According to this understanding, oxygen atoms at the surface and an atomic structure without strain are important requirements for large gain. In this sense, other crystal structures of $\mathrm{SiO}_{2}$ should also produce large gain if the strain at the interface is small.

It is worth mentioning that all $M_{\|}(\boldsymbol{r})$ that do not vanish at the integration [Figs. 4(c), 4(h), 4(j), and 4(k)] have a layered structure where each layer consists of rugbyball-shaped isosurfaces whose height is two silicon layers. The number of rugbyball layers can thus be expressed as $l=(i-1) / 2$, where $i$ is the number of silicon layers. Provided that the sign of $M_{\|}(\boldsymbol{r})$ is changed by such layers alternately, the optical matrix element will have relatively large values when $l$ is odd, i.e., $i=3,7,11 \ldots$.

Because the calculations in Fig. 3 are only for gapcrossing transitions, we have to take account transitions within VBs and those within CBs such as free-carrier absorptions, to determine whether the gains remain positive or not. To do so, DFT's underestimation of energy gap should be corrected because the energy dependence of the positive contributions and the negative contributions to the gain are inconsistent. For that purpose, CBs of a silicon film are uniformly shifted ${ }^{21}$ by $+0.44 \mathrm{eV}$. The calculated band gap of the bulk silicon agrees with experiment when this value is added. After that correction, gain spectra of seven-atomiclayer silicon film between quartz layers are calculated taking account of all possible transitions. As a result, we obtained a positive optical gain. This means that an ultrathin silicon film has a positive optical gain due to an intrinsic quantum confinement effect.

Enhancement of light emission by excitons ${ }^{22}$ is not considered here because the carrier density we are discussing is very high. Excitons should be taken into account, however, even in such carrier densities when the gains are close to zero.

Figure 5 shows maximum optical gains of the silicon film

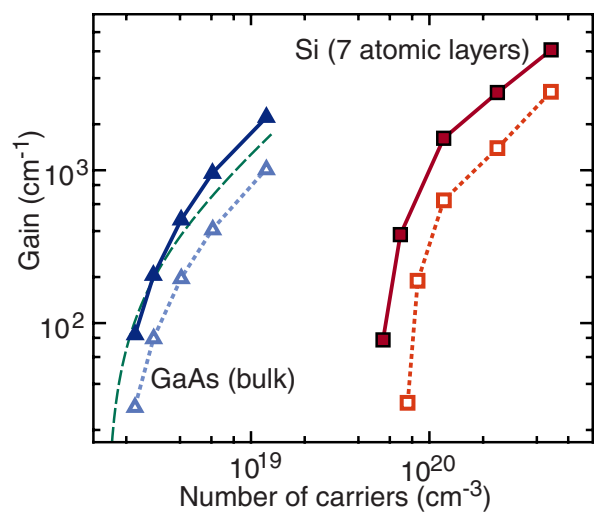

FIG. 5. (Color online) Maximum optical gains of bulk GaAs and a silicon film between quartz as a function of injected carrier density. Solid lines and dotted lines show the gains calculated taking account of gap-crossing transitions only and all transitions, respectively. Dashed line shows an experimental result of bulk GaAs (Ref. 23). 
as a function of carrier density. The dotted line and the solid line show the gains with and without free-carrier absorption. Those of the bulk GaAs calculated in the same manner are also shown in the figure, together with a dashed line fitted to experimental values. ${ }^{23}$ It is clear that the gain of the silicon film is smaller than that of the GaAs by an order or so, if it is compared at the same carrier density after extrapolation. In an ultrathin film, minimum carrier density necessary to reach population inversion is higher than that in a bulk crystal due to the step-functionlike rising edge of the two-dimensional density of states. However, it can be said also that the gain of the silicon film is comparable to that of bulk GaAs if the carrier density is ten times larger than that of GaAs. Actually, it is well known that the maximum carrier density practicable in silicon devices is much larger than that in GaAs devices due to the higher solid solubility limit of impurities in silicon.

In summary, we calculated optical gains of ultrathin $\mathrm{Si}$ films from first principles and found that the net optical gain can be positive because of an intrinsic origin. These films have direct bandgap structures due to the quantum confinement effects such as the valley projection and the rise of LCB at M point. Enhancement of light emission due to an appropriate surface structure of silicon is also important . $\mathrm{SiO}_{2}$ crystal that forms a strainless connection with a $\mathrm{Si}(001)$ surface such as quartz, fairly enhances optical gain. Calculated gain of such a film is comparable to that of the bulk GaAs if the injected carrier density is large enough. Therefore, we predict that lasing in such an ultrathin silicon film is achievable when the cavity suitable for induced emission is prepared.

We would like to thank H. Kageshima, J. Yamauchi, and Y. Yoshimoto for valuable discussions on the calculation methods. The first-principle calculations were performed with TAPP (the Tokyo Ab initio Program Package).
${ }^{1}$ S. Ossicini, L. Pavesi, and F. Priolo, Light Emitting Silicon for Microphotonics (Springer, New York, 2004).

${ }^{2}$ L. T. Canham, Appl. Phys. Lett. 57, 1046 (1990).

${ }^{3}$ L. Pavesi, L. Dal Negro, C. Mazzoleni, G. Franzò, and F. Priolo, Nature (London) 408, 440 (2000).

${ }^{4}$ D. J. Lockwood, Z. H. Lu, and J.-M. Baribeau, Phys. Rev. Lett. 76, 539 (1996).

${ }^{5}$ E.-C. Cho, M. A. Green, R. Corkish, P. Reece, M. Gal, and S.-H. Lee, J. Appl. Phys. 101, 024321 (2007).

${ }^{6}$ For recent progress, see, e.g., Fifth International Conference on Group IV Photonics (IEEE, New York, 2008).

${ }^{7}$ H. Chen, J. H. Shin, P. M. Fauchet, J.-Y. Sung, J.-H. Shin, and G. Y. Sung Appl. Phys. Lett. 91, 173121 (2007).

${ }^{8}$ S. Saito, D. Hisamoto, H. Shimizu, H. Hamamura, R. Tsuchiya, Y. Matsui, T. Mine, T. Arai, N. Sugii, K. Torii, S. Kimura, and T. Onai, Jpn. J. Appl. Phys. 45, L679 (2006); Appl. Phys. Lett. 89, 163504 (2006).

${ }^{9}$ J. P. Perdew, K. Burke, and Y. Wang, Phys. Rev. B 54, 16533 (1996).

${ }^{10}$ D. Vanderbilt, Phys. Rev. B 41, 7892 (1990).

${ }^{11}$ K. Laasonen, A. Pasquarello, R. Car, C. Lee, and D. Vanderbilt,
Phys. Rev. B 47, 10142 (1993).

${ }^{12}$ H. Kageshima and K. Shiraishi, Phys. Rev. B 56, 14985 (1997).

${ }^{13}$ L. J. Sham, S. J. Allen, A. Kamgar, and D. C. Tsui, Phys. Rev. Lett. 40, 472 (1978).

${ }^{14}$ T. Ohno, K. Shiraishi, and T. Ogawa, Phys. Rev. Lett. 69, 2400 (1992).

${ }^{15}$ R. Buczko, S. J. Pennycook, and S. T. Pantelides, Phys. Rev. Lett. 84, 943 (2000).

${ }^{16}$ Y. Tu and J. Tersoff, Phys. Rev. Lett. 84, 4393 (2000).

${ }^{17}$ E.-C. Cho, M. A. Green, J. Xia, R. Corkish, and A. Nikulin, J. Appl. Phys. 96, 3211 (2004).

${ }^{18}$ M. Watarai, J. Nakamura, and A. Natori, Phys. Rev. B 69, 035312 (2004).

${ }^{19}$ S. L. Chuang, Physics of Optelectronic Devices (WileyInterscience, New York, 1995).

${ }^{20}$ M. Nishida, Phys. Rev. B 58, 7103 (1998).

${ }^{21}$ S. N. Rashkeev, W. R. L. Lambrecht, and B. Segall, Phys. Rev. B 57, 3905 (1998).

${ }^{22}$ L. V. Keldysh, Phys. Status Solidi A 164, 3 (1997).

${ }^{23}$ A. Yariv and P. Yeh, Photonics, 6th ed. (Oxford University Press, New York, 2007). 